\title{
WISP2 promotes cell proliferation via targeting ERK and YAP in ovarian cancer cells
}

\author{
Zi-Qing Shi ${ }^{\dagger}$, Zi-Yan Chen ${ }^{\dagger}$, Yao Han, Heng-Yan Zhu, Meng-Dan Lyu, Han Zhang, Yi Zhang, Liu-Qing Yang and \\ Wei-Wei Pan ${ }^{*}$ (i)
}

\begin{abstract}
Background: Wnt-inducible signaling pathway protein 2 (WISP2) is a wnt1-induced signaling pathway protein 2. Although studies indicate that WISP2 may promote the development of various tumors, its role in ovarian cancer remains unclear. The objective of the current study was to analyze the effects of WISP2 on the proliferation and migration of ovarian cancer cells in vitro and in vivo.

Results: Immunohistochemistry and western blotting indicated that WISP2 was highly expressed in various ovarian cancer tissues and cell lines, but weakly expressed in normal ovary tissue. WISP2 deletion inhibited cell growth, clone formation, and migration of ovarian cancer cells while promoting cell apoptosis and affecting the cell cycle. This growth inhibitory effect caused by WISP2 loss is due to the inhibition of phosphorylated extracellular signalrelated kinase (p-ERK)1/2, as well as CCAAT/enhancer-binding protein a (CEBPa) and CEPBß. In addition, WISP2 deletion also activated the Yes-associated protein (YAP).
\end{abstract}

Conclusion: WISP2 deletion inhibits ovarian cancer cell proliferation by affecting ERK signaling pathways.

Keywords: Ovarian cancer, Proliferation, Apoptosis, WISP2

\section{Background}

Epithelial ovarian cancer (EOC) is the fifth leading cause of all female cancer-related deaths in the USA, with approximately 22,000 new cases and 16,000 deaths per year. It is the most prevalent and lethal of all gynecologic cancers [1]. Primary treatment includes surgery and platinum-based chemotherapy. In spite of treatments such as ovarian cytoreductive surgery combined with effective postoperative chemotherapy, prognosis remains unsatisfactory, and mortality statistics have changed only slightly [2]. Therefore, molecular target screening of ovarian cancer is felt to be particularly important.

\footnotetext{
* Correspondence: wwpan@mail.zjxu.edu.cn

${ }^{\dagger}$ Zi-Qing Shi and Zi-Yan Chen are co-first authors

School of Medicine, Jiaxing University, Jiaxing 314001, China
}

Wnt-inducible signaling pathway protein 2(WISP2)is a member of the $\mathrm{CCN}$ protein family, which comprises six members, including CCN1 (CYR61), CCN2 (CTGF), CCN3 (NOV), CCN4 (WISP1), CCN5 (WISP2), and CCN6 (WISP3) [3-5]. Previous studies have revealed that CCNs exhibit different expression profiles and transcript levels in different tissues, organs, and tumors [6]. The six CCN members are associated with numerous cellular functions as well as pathological conditions [7]. WISP2 is a member of the cysteine-rich CCN family of extracellular matrixassociated proteins [8]. Emerging studies have demonstrated that, in cancers, WISP2 is involved in cell proliferation, migration, and metastasis [9]. However, the function of WISP2 in ovarian cancer cells remains largely unclear.

Our previous studies have indicated that WISP2 is induced by LATS1/2 deletion in MC38 cells and affected

C C The Author(s). 2020 Open Access This article is licensed under a Creative Commons Attribution 4.0 International License, which permits use, sharing, adaptation, distribution and reproduction in any medium or format, as long as you give appropriate credit to the original author(s) and the source, provide a link to the Creative Commons licence, and indicate if changes were made. The images or other third party material in this article are included in the article's Creative Commons licence, unless indicated otherwise in a credit line to the material. If material is not included in the article's Creative Commons licence and your intended use is not permitted by statutory regulation or exceeds the permitted use, you will need to obtain permission directly from the copyright holder. To view a copy of this licence, visit http://creativecommons.org/licenses/by/4.0/ The Creative Commons Public Domain Dedication waiver (http://creativecommons.org/publicdomain/zero/1.0/) applies to the data made available in this article, unless otherwise stated in a credit line to the data. 
cell growth and apoptosis [10]. In the current study, we found that WISP2 was highly expressed in various ovarian cancer tissues and cell lines but weakly expressed in human normal ovary tissue. To determine the role of WISP2 in ovarian cancer, we deleted WISP2 using the CRISPR/ Cas9 method. WISP2 deletion inhibited the growth, clone formation, and cell migration of ovarian cancer cells. Furthermore, WISP2 deletion promoted cell apoptosis and affected the cell cycle. These growth inhibitory effects of WISP2 loss were due to the inhibition of the phosphorylated extracellular signal-related kinase (p-ERK)1/2, as well as CCAAT/enhancer-binding protein $\alpha(\mathrm{CEBP} \alpha)$ and CEBP $\beta$. In addition, WISP2 deletion also activated the Yes-associated protein (YAP). The current study revealed the potential of WISP2 as a factor capable of promoting ovarian cancer cell proliferation and survival.

\section{Results}

WISP2 is overexpressed in human ovarian cancer tissues and cell lines

WISP2 protein expression in human normal ovary tissues and ovarian cancer tissues was assessed via immunohistochemistry. WISP2 was weakly expressed in normal ovarian tissue $(n=3)$. Significant WISP2 staining intensity was detected in endometrioid carcinoma, serous cystadenoma, clear cell carcinoma, and mucinous cystadenoma tissues $(n=20)$ (Fig. 1a and b). The expression of WISP2 in ovarian cancer cell lines and immortalized ovarian surface epithelial (mOSE) cells was also examined. WISP2 was highly expressed in most ovarian cancer cell lines, such as ES-2 and HO8910 (Fig. 1c), but was weakly expressed in mOSE cells. High expression of WISP2 protein in ovarian cancer tissues and cell lines suggest that WISP2 may play an essential role in ovarian tumor cell proliferation and migration.

\section{WISP2 deletion inhibits cell growth and cell migration}

To verify this assumption, we deleted WISP2 in the ovarian cancer cell lines ES-2 and HO8910 using CRISPR/ Cas9 technology. By evaluating the protein expression of WISP2, we confirmed the successful generation of complete WISP2 knockout (KO) clones from the ES-2 cell line. However, it was somewhat difficult to obtain complete WISP2 KO clones from HO8910 cells (Fig. 1d).

We tested whether knocking out WISP2 inhibits the growth of ovarian cancer cells. For this reason, we compared the cell growth between wild-type (WT) and WISP2 KO cells by cell growth assay. WISP2 deletion significantly suppressed cell growth in ES-2 and HO8910 cells (Fig. 2a). We also examined anchorage-independent growth of WT and WISP2 KO cells via a soft agar assay and found that WISP2 deletion strongly inhibited anchorage-independent growth in ES-2 and HO8910 cells (Fig. 2b). Next, we analyzed the expression of the cancer cell proliferation markers KI-67 and p-Histone $\mathrm{H} 3$ using immunofluorescence and western blot assay. WISP2 deletion significantly inhibited KI-67 and pHistone H3 protein expression in ES-2 and HO8910 cells (Fig. 4a and b).

The effect of WISP2 on ovarian cancer cell migration was also determined. A scratch wound assay demonstrated that WISP2 deletion noticeably inhibited the migration of ES-2 cells (Fig. 2c). In addition, we examined the effect of WISP2 deletion on the metastatic potential of ovarian cancer ES-2 and HO8910 cells using a transwell assay. The migration of the cells was decreased upon WISP2 deletion (Fig. 2d). These results demonstrate that WISP2 is required for ovarian cancer cell proliferation and migration.

\section{WISP2 deletion promotes apoptosis and senescence}

FACS analysis revealed that WISP2 KO cells were arrested in the G2 phase of the cell cycle (Fig. 3a). Furthermore, we examined apoptosis of WISP2 deleted cells using a PE Annexin $\mathrm{V}$ apoptosis detection kit. Apoptosis was increased in WISP2 KO cells (Fig. 3b). In addition, staining with the senescence marker senescence-associated $\beta$ galactosidase (SA- $\beta$-gal) confirmed that WISP2 deleted cells displayed elevated SA- $\beta$-gal staining (Fig. 3c). These data indicate that WISP2 deletion results in cellular senescence. Furthermore, we analyzed the p16/pRB axes and $\mathrm{p} 53$, which are major senescence-triggering pathways [11]. We found that the levels of $p 16, M d m 2$ and Perp were increased in WISP2 deleted cells, verifying that knockout of WISP2 induces cellular senescence (Fig. 3d).

\section{WISP2 deletion inhibits cell proliferation by affecting the ERK signaling pathway}

To examine the mechanisms underlying the role of WISP2 in ovarian cancer cell growth regulation, we examined the cell proliferation marker KI-67 and the cell apoptosis marker cleaved caspase-3 via immunofluorescence assays. The results showed that WISP2 deletion significantly inhibited KI-67 expression in the nucleus and promoted apoptosis (Fig. 4a). Western blot analysis confirmed that the reduction in WISP2 protein expression inhibited p-ERK1/2, CEBP $\alpha$, and CEBP $\beta$. Furthermore, we observed that the deletion of WISP2 inhibited YAP phosphorylation (Fig. 4b) and increased the expression of the DNA damage marker p-H2AX. q-PCR analysis showed that the expression of the YAP target genes Ctgf, Cyr61, Ankrd, and Amotl2 were increased in WISP2 KO cells (Fig. 5a). In addition, the expression of the ERK1/2 target genes $c-M y c, E L K-1$, and P90RSK also increased in WISP2 deletion cells, while that of the extracellular matrix-associated genes $\mathrm{Mmp} 13$ and snail significantly decreased. Considering all of these findings, we propose that WISP2 deletion in ovarian cancer cells 


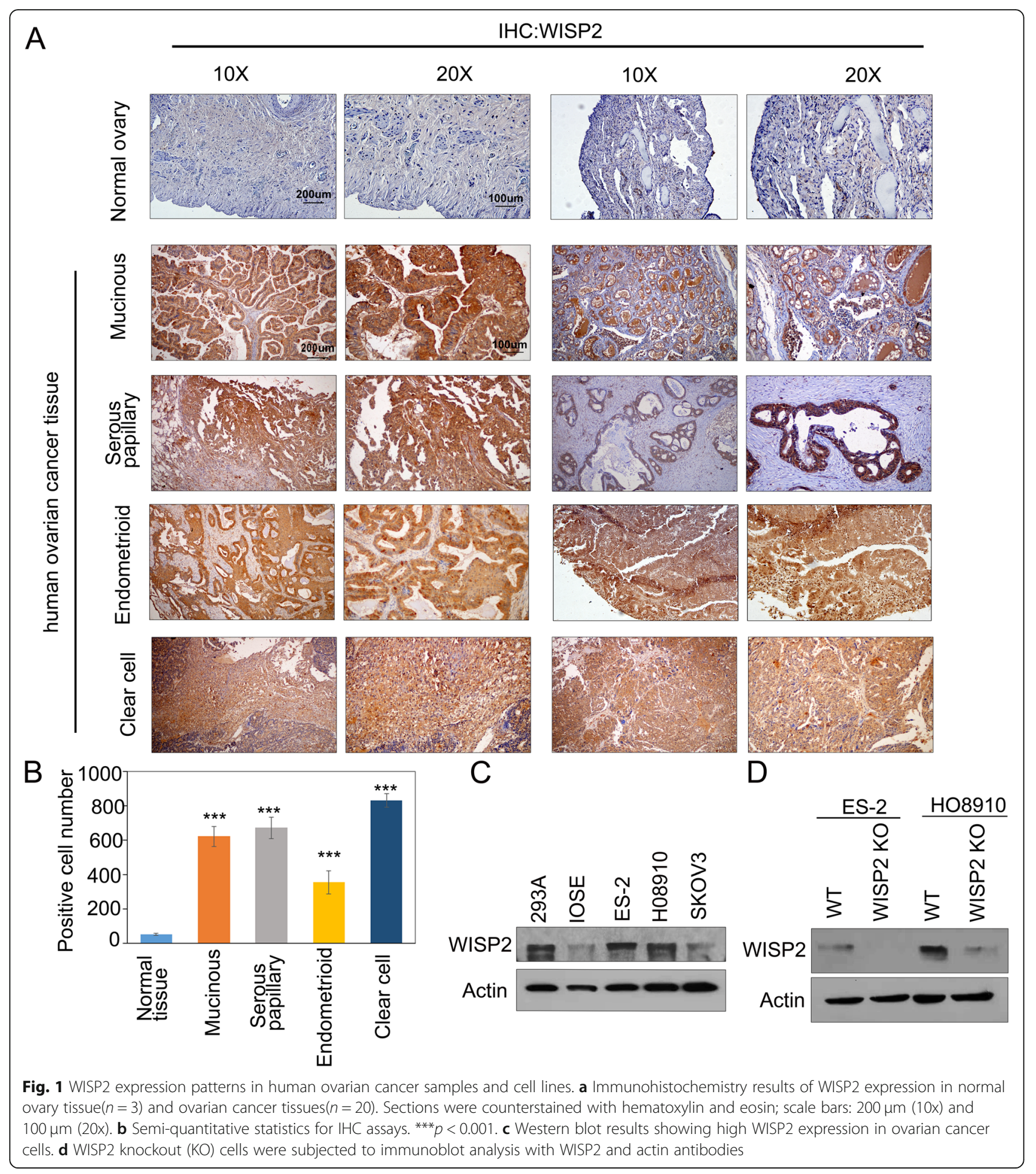

represses cell proliferation and increases senescence as well as apoptosis by affecting the ERK and Hippo signaling pathways.

\section{WISP2 deficiency inhibits tumor growth in vivo}

To evaluate the role of WISP2 in ES-2 tumor growth, we performed xenograft experiments in nude mice. Tumors grew slower in mice transplanted with WISP2 deficient ES2 cells than in control mice (ES-2 WT group) (Figs. 6a and b). q-PCR results showed that the YAP (Cyr61, Amotl2, and $A n k r d 1)$ and ERK1/2 target genes (c-Myc and ELK-1) were elevated in WISP2 deficient tumor samples (Fig. 6c). In addition, we found that the level of cleaved caspase- 3 was increased, whereas that of p-Histone H3, p-ERK1/2, 
A

A

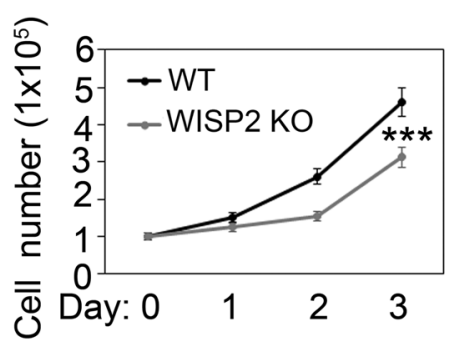

B
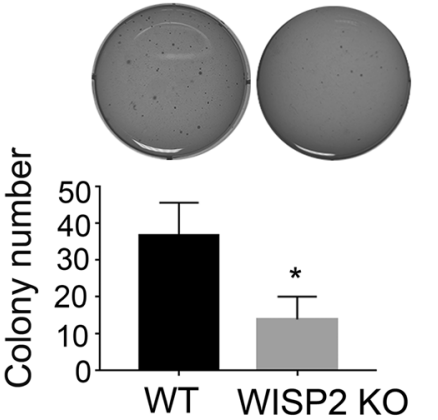

C
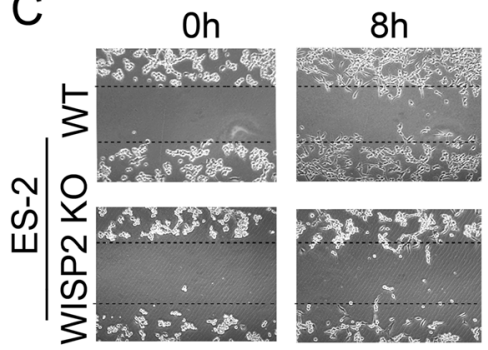

$20 \mathrm{~h}$

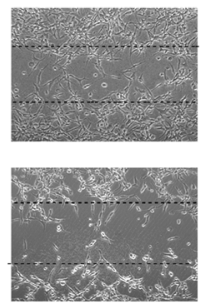

HO8910

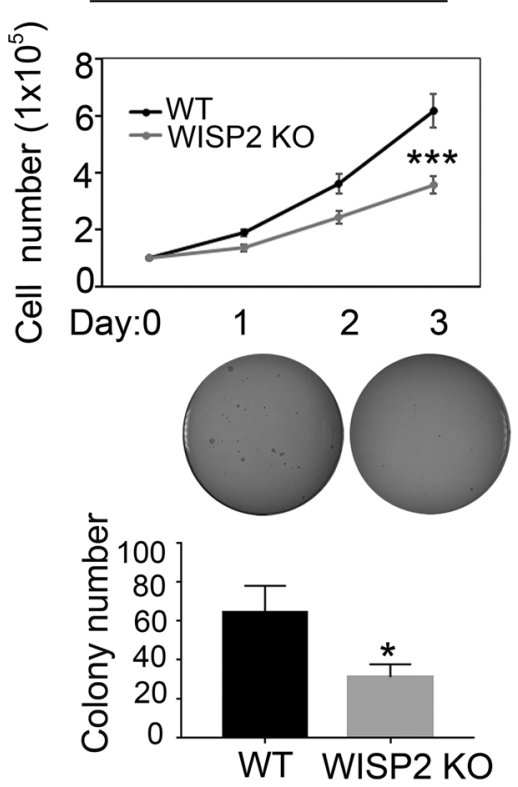

\section{D}
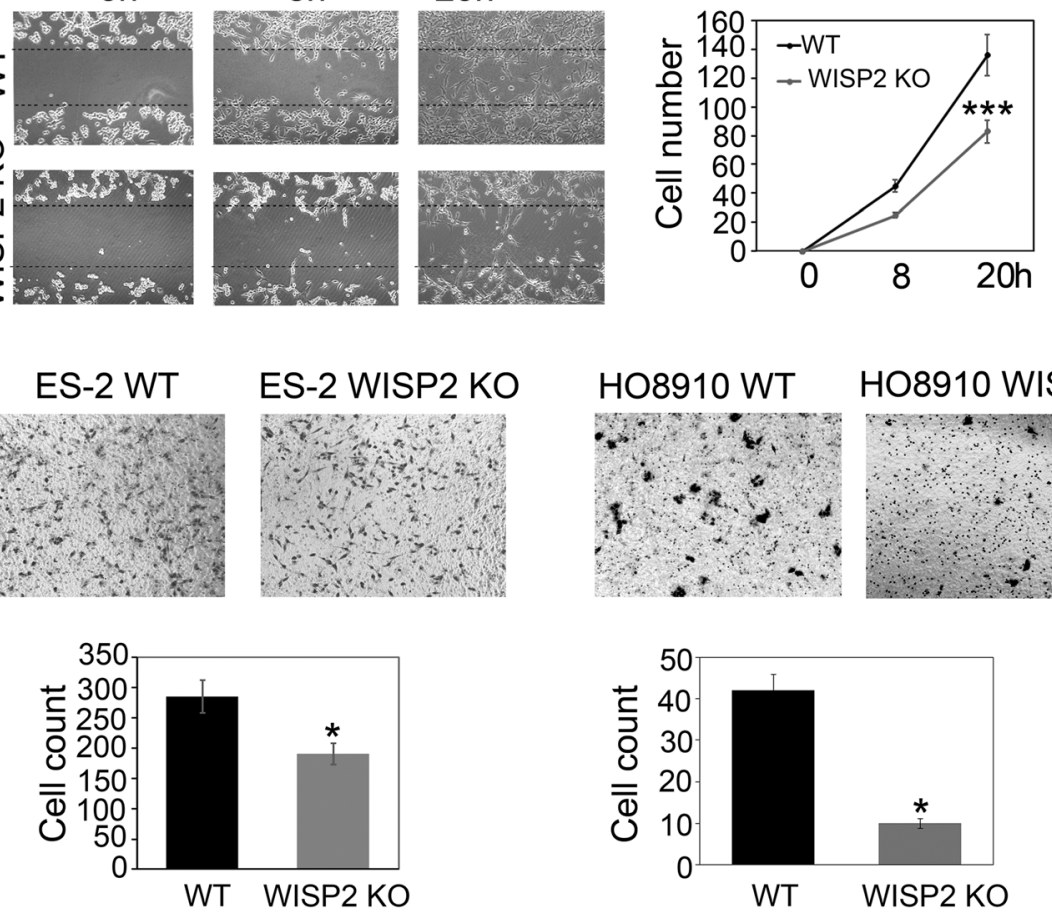

Fig. 2 WISP2 deletion represses cell proliferation and migration. a WISP2 deletion inhibits ovarian cancer cell growth. ES-2 and HO8910 WT and WISP2 deletion cells $\left(1 \times 10^{5}\right)$ were plated in 6-well culture dishes, and viable cells were counted following trypan blue staining. $\mathbf{b}$ WISP2 deletion inhibits anchorage-independent growth of ES-2 and HO8910 cells. Cells were cultured in Matrigel. Colonies that formed on days 12 and 17 are shown. Colony numbers were counted on days 14 to 21. c Wound-healing assay for the migration capability of ES-2 WT and WISP2 deletion cells. d Transwell test to detect the migration capability of ES-2 and HO8910 WT and WISP2 deletion cells. Cells were added to transwells and allowed to migrate for $12 \mathrm{~h}$. Cells at the upper surface of the membrane were removed with cotton swabs, and cells on the bottom surface were stained with hematoxylin and eosin. The experiment was replicated thrice. Error bars represent the standard deviation (s.d.). ${ }^{*} p<0.05$,

${ }^{* *} p<0.01,{ }^{* * *} p<0.001$ 


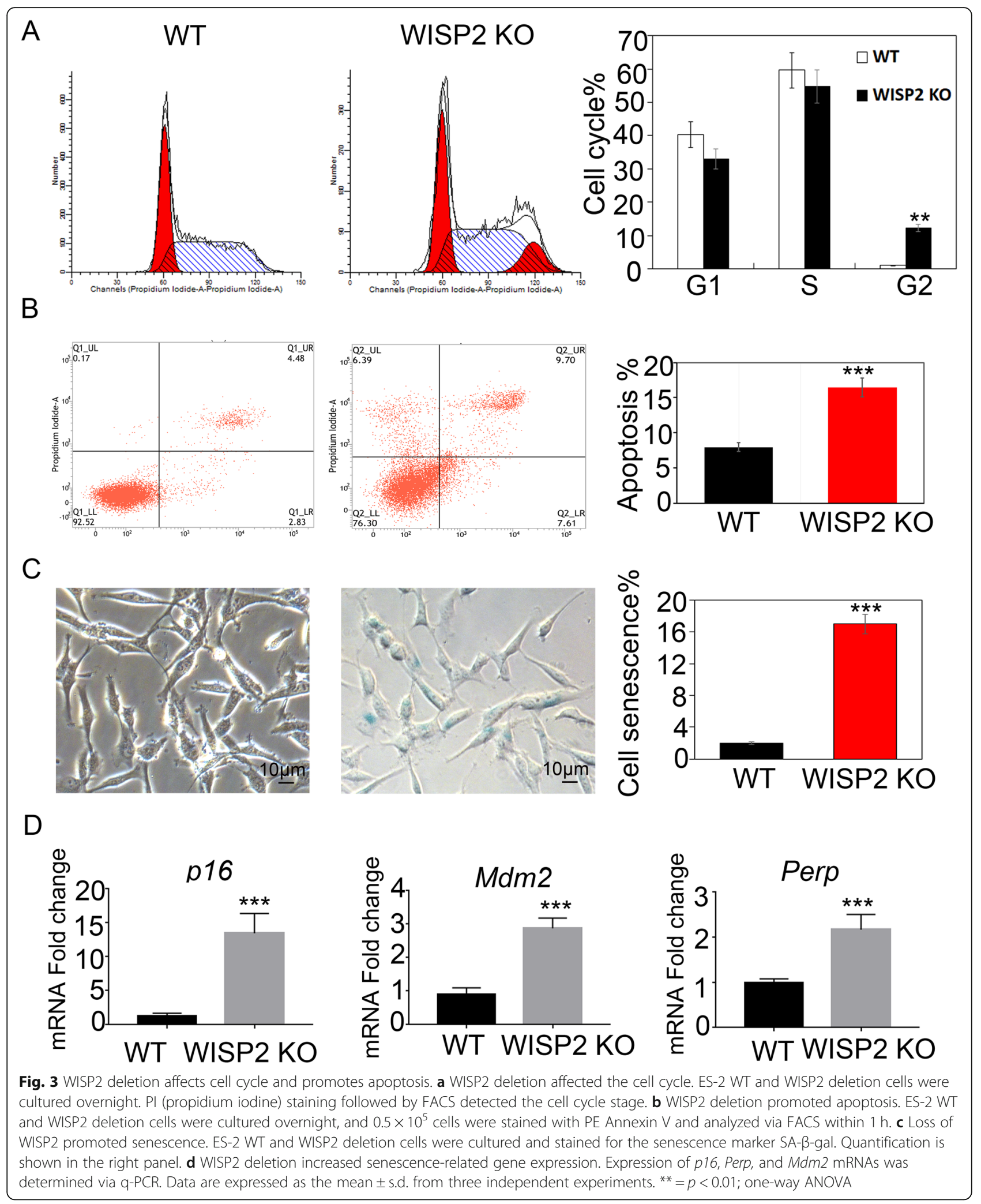


A
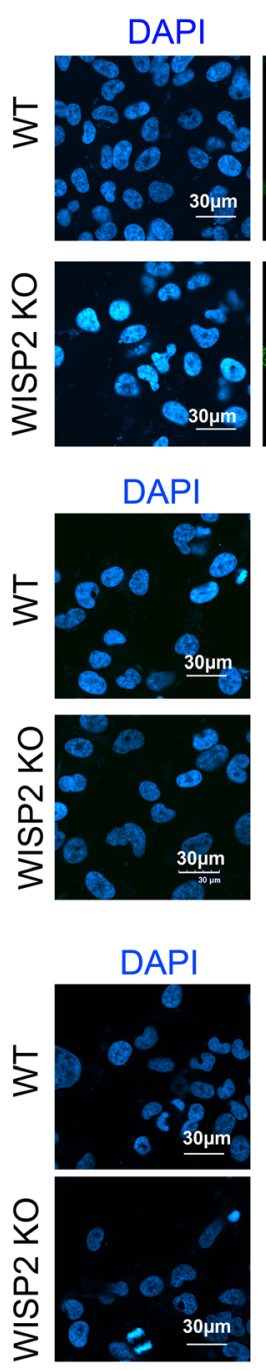

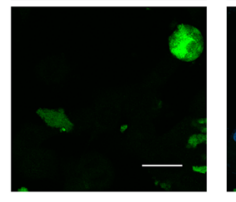

$p-H 2 A X$
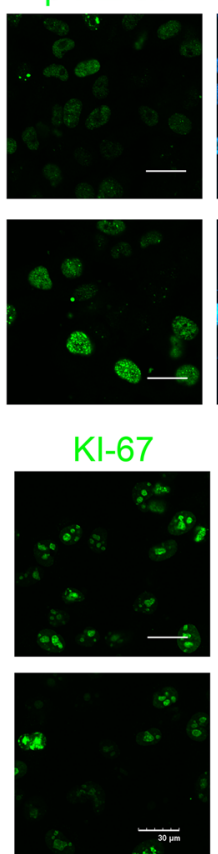

Cleaved

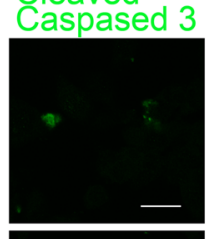

Merge
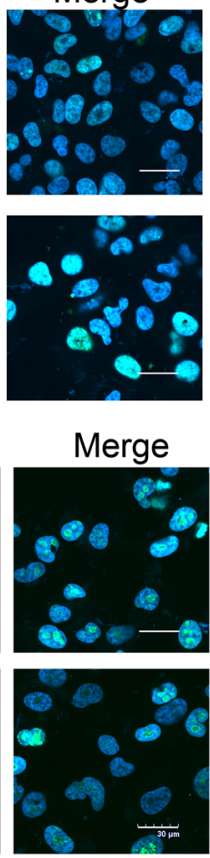

Merge
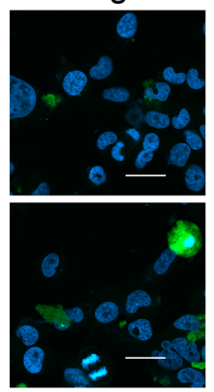

B

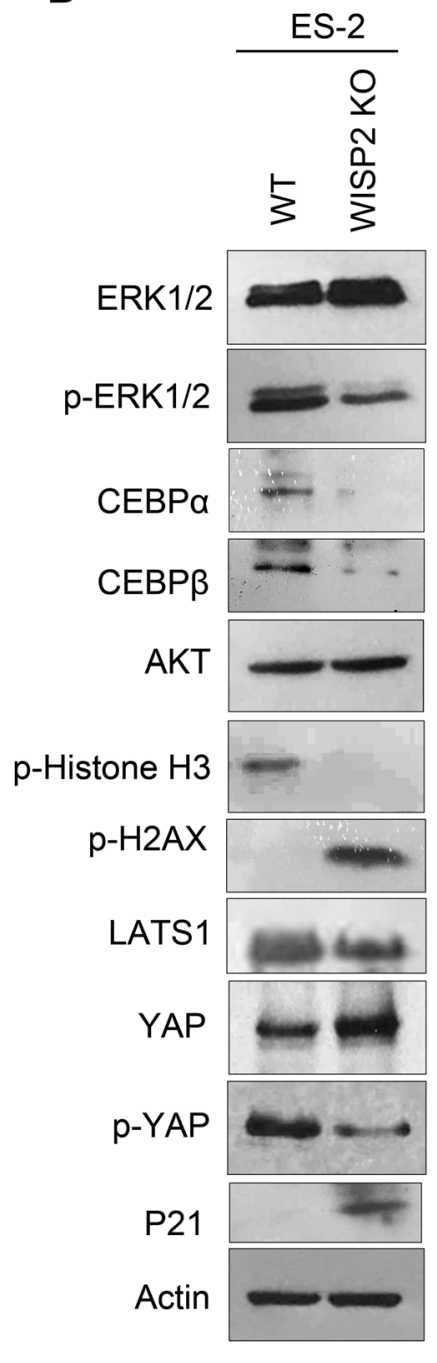

Fig. 4 WISP2 deletion affects the ERK1/2 and Hippo signaling pathways. a WISP2 deletion inhibited the expression of the proliferation marker KI67 and increased that of the apoptosis marker cleaved caspase-3. b ES-2 WT and WISP2 deletion cells were cultured for $24 \mathrm{~h}$. Western blot was performed with the indicated antibodies

and p-YAP were decreased in WISP2 deficient tumors (Fig. 6d). Western blotting confirmed the reduction in WISP2 protein, YAP S127 phosphorylation, and ERK1/2 phosphorylation in tumors derived from WISP2 KO cells (Fig. 6e). Collectively, our results indicate that WISP2 deletion inhibits ovarian cancer cell growth in vitro and in vivo.

\section{Discussion}

WISP2 reportedly governs the expression of several genes in human cancer cells, such as those of breast, colon, gastric, pancreatic, and esophageal cancers [1215]. Clinical studies have shown that the expression profile and role of WISP2 in various cancers may differ, and such inconsistencies, which have been observed between multiple cancers, have raised uncertainty concerning the role of WISP2 in carcinogenesis. For example, in MCF-7 cells, a proliferative role of WISP2 has been reported in which silencing WISP2 inhibited cell proliferation induced by serum and epidermal growth factor, whereas it acts as a growth repressor in prostate cancer cells [16, 17]. In addition, WISP2 likely plays a preventive role in the progression of pancreatic cancer by participating in morphological alterations from mesenchymal to epithelial transition (MET) of pancreatic adenocarcinoma [12] and breast cancer cells [18]. However, the role of WISP2 in ovarian cancer cells remains unelucidated.

The present study indicates that although WISP2 protein levels were elevated in different ovarian cancer tissues and cell lines, it was minimally detectable in normal ovarian epithelial cells. This suggests that elevated 


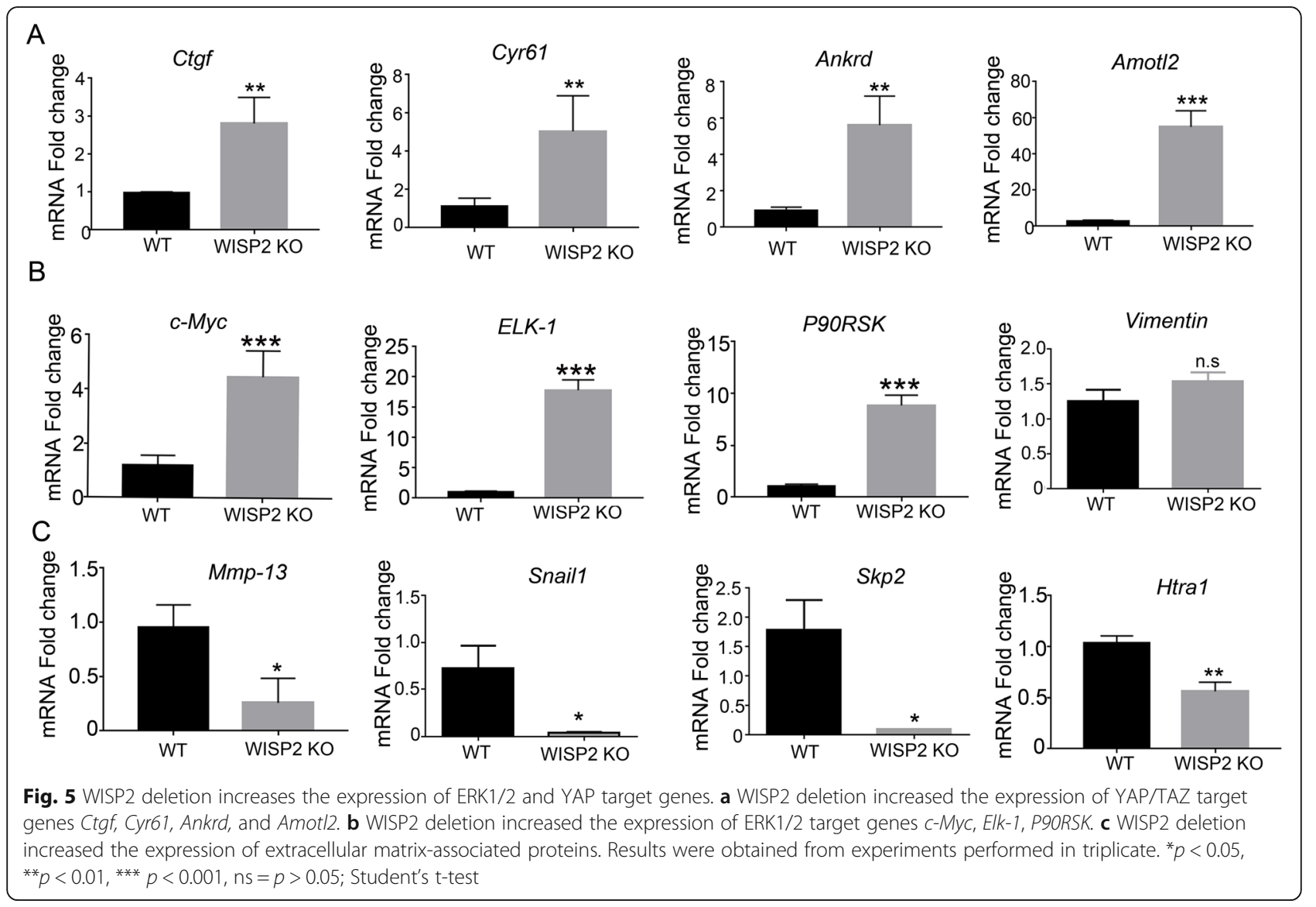

WISP2 levels may play a specific role in human ovarian cancer progression. Deletion of WISP2 inhibited ovarian cancer cell proliferation and clone number and induced cell apoptosis as well as senescence. Molecular mechanism studies have indicated that WISP2 deletion inhibited ERK1/2 and YAP phosphorylation in vivo and in vitro and subsequently increased p16 and p21 accumulation.

The mitogen-activated protein kinase/ERK (MAPK/ERK) and Hippo pathways are reportedly associated with cell proliferation, differentiation, migration, senescence, and apoptosis $[19,20]$. Previous studies have shown that alteration in the MAPK/ERK pathway is strongly implicated in ovarian cancer pathogenesis [21, 22]. Dang et al. [23] reported that metformin combined with cisplatin inhibited cell viability and promoted apoptosis of human ovarian cancer cells by inactivating ERK1/2. Xia et al. [24] reported that YAP, which promotes ovarian cancer cell tumorigenesis, was indicative of a poor prognosis for ovarian cancer patients. A previous study of ours demonstrated that the deathassociated protein 6 (DAXX) could induce ovarian cancer ascites formation by activating the ERK signaling pathway [25]. Large tumor suppressor (LATS)1/2 deletion inhibited colon cancer cells by promoting YAP transcription [10]. In the current study, we found that WISP2 deletion inhibited the expression of p-ERK and p-YAP, which induced the expression of ERK1/2 and YAP target genes, such as Ankrd, Amotl2, c-Myc, ELK-1, and p90RSK. Thus, we concluded that the effect of WISP2 inhibition on ES-2 and H08910 cells may, at least partly, be due to the downregulation of $\mathrm{p}$-ERK $1 / 2$ and $\mathrm{p}$-YAP.

In addition, WISP2 was reported as an extracellular matrix (ECM)-associated adhesion protein as it contains binding sites for both ECM and cell surface proteins. WISP2 regulated cell growth, migration, and metastasis by regulating EMT and inhibiting matrix metalloproteinase (MMP)-9 and MMP-2 via ERK in gastric cancer cells [26]. In the present study, WISP2 deletion decreased the expression of p-ERK1/2. Thus, WISP2 may inhibit the proliferation and induce apoptosis by suppressing ERK1/ 2 signaling in ovarian cancer cells. E-cadherin is a key molecule involved in EMT and cell invasion [27, 28]. WISP2 regulated cell migration and invasion by affecting Snail and E-cadherin in breast cancer cells [29]. Our study also demonstrated that WISP2 deletion decreased Snail and MMP-13 levels in ovarian cancer cells. Therefore, WISP2 may promote invasion and migration by regulating EMT in ovarian cancer cells.

Our data on WISP2 expression in ovarian cancer cells have clinical and therapeutic implications. WISP2 deletion suppressed the tumorigenic capacity of ovarian cancer 


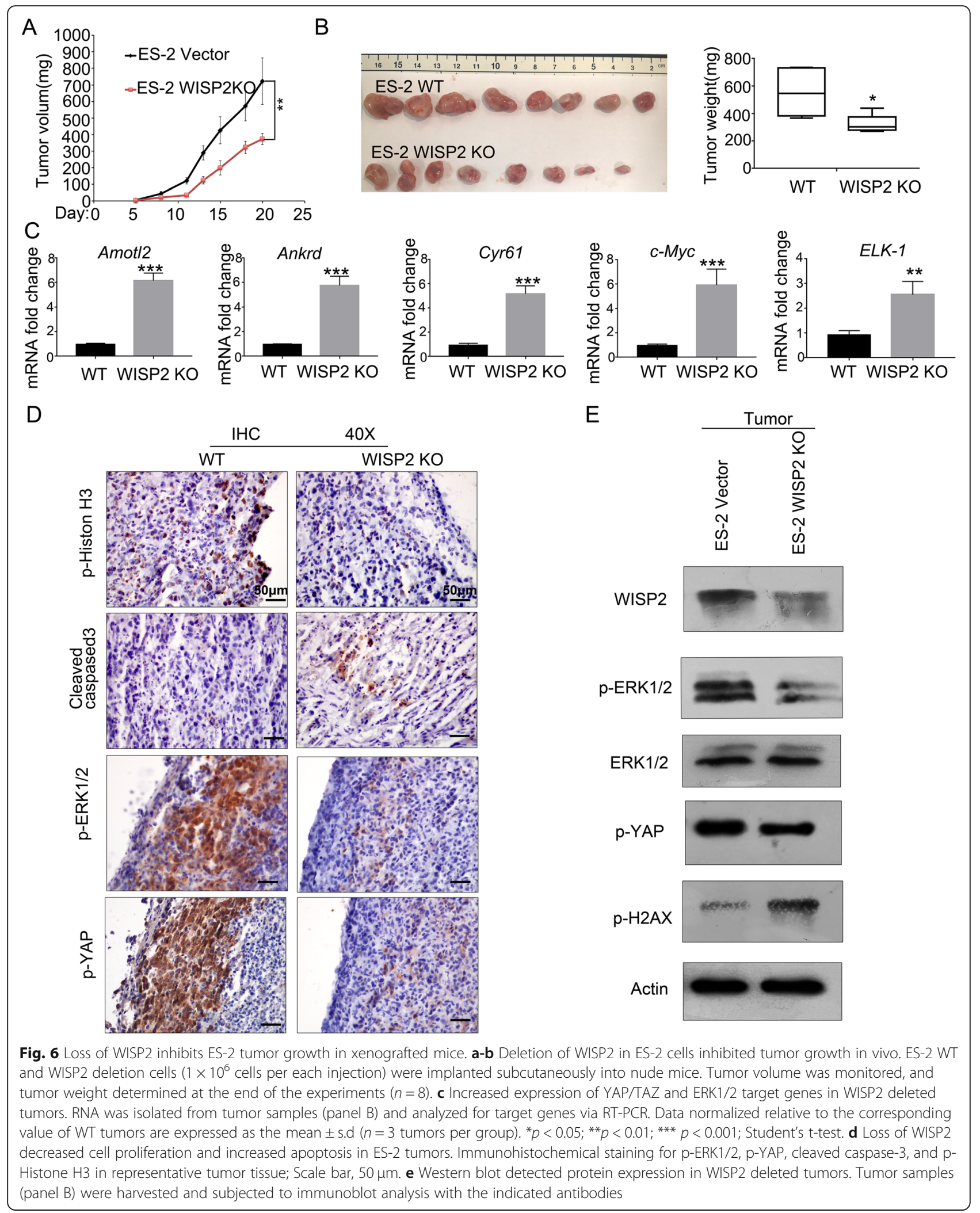


cells in vivo and in vitro. Thus, small molecules capable of inhibiting WISP2 activity or accumulation may have therapeutic potential in ovarian cancer patients. These results indicate that WISP2 expression may play a key role in initiating ovarian cancer and, therefore, be used as an early diagnostic marker for ovarian cancer.

\section{Conclusions}

WISP2 deletion inhibits ovarian cancer cell proliferation by affecting ERK and YAP signaling pathways.

\section{Methods}

\section{Cell culture and CRISPR}

HO8910 and ES-2 ovarian cancer cells were cultured in DMEM/RPMI1640 supplemented with 10\% FBS and 1\% penicillin-streptomycin solution at $37^{\circ} \mathrm{C}$ under a humidified $5 \% \mathrm{CO}_{2}$ atmosphere.

WISP2 deletion cells were generated via CRISPR genomic editing technology. These plasmids were then transfected into HO8910 and ES-2 cells. Twenty-four hours following transfection, transfected cells were enriched via puromycin selection for $3 \mathrm{~d}$ and sorted on 96-well plates with only a single cell per well. The clones were screened via western blot using WISP2 antibody (1: 2000, Abcam).

Wisp2 sgRNA sequence was as follows:

Wisp2-1: GCTGTGAGGTGAATGGCCGC.

Wisp2-2: TTGCCGGCTGCATCACTGCC.

\section{Soft agar colony formation assay}

One-milliliter layers of $0.5 \%$ agar were prepared in a 35 $\mathrm{mm}$ cell culture dish. Cells were suspended in $1 \mathrm{ml}$ of $0.35 \%$ agar containing $1 \times$ cell culture medium and $10 \%$ FBS and poured over these layers. The final cell concentration in each culture was $0.5 \times 10^{3}$ cells $/ \mathrm{ml}$. Triplicate cultures were used for each experiment. Plates were placed in a $5 \% \mathrm{CO}_{2}$ humidified incubator at $37^{\circ} \mathrm{C}$. Colonies were counted 2-3 weeks after plating using an Omnicon FAS II Image Analysis System.

\section{Wound-healing assay}

ES-2 WT and WISP2 deleted cells were grown in DMEM supplemented with 10\% FBS until confluence was reached. The medium was then changed to fresh serum-free medium, and the cell monolayers were scraped in a straight line using a P-10 pipette tip to create a scratch. The plates were photographed at 0 and $24 \mathrm{~h}$ using a phase-contrast inverted microscope (Nikon Ti, Nikon Corp.).

\section{Transwell migration assay}

Twenty-four-well tissue culture plate inserts with $8 \mu \mathrm{m}$ pore filters and BioCoat Matrigel (BD Biosciences, Bedford, MA, USA) were used to assess the migration and invasive potential of ES-2 and HO8910 WT and WISP2 deleted cells. The cells were suspended in serum-free medium and then added to a transwell $(100 \mu \mathrm{l}$ cell suspension/well at a concentration of $0.5-1 \times 10^{5}$ cells $/ \mathrm{ml}$ ). After incubation for $24 \mathrm{~h}$ at $37^{\circ} \mathrm{C}$, cells at the upper surface of the transwell were removed using cotton swabs. Migrated cells that had attached to the lower surface were stained using hematoxylin and eosin. Transwells were rinsed with water and air-dried. Positive cells were quantified using Image-Pro Plus 6.0 software.

\section{PE Annexin V apoptosis detection}

ES-2 WT and WISP2 deficient cells were cultured in 6well plates overnight. Cells were washed twice with cold PBS and resuspended in $1 \times$ Binding Buffer at a concentration of $1 \times 10^{6}$ cells $/ \mathrm{ml}$. Next, $1 \times 10^{5}$ cells were transferred to a $5 \mathrm{ml}$ culture tube, treated with $5 \mu \mathrm{l}$ of $\mathrm{PE}$ Annexin $\mathrm{V}$ and 7-amino-actinomycin $\mathrm{D}$, gently vortexed, and incubated for $15 \mathrm{~min}$ at $25^{\circ} \mathrm{C}$ in the dark. After incubation, $400 \mu \mathrm{l}$ of $1 \times$ Binding Buffer was added to each tube, and cells were analyzed via flow cytometry within $1 \mathrm{~h}$.

\section{Mice and xenograft models}

Mice $(n=8)$ were housed under standard conditions with a $14 \mathrm{~h} / 10 \mathrm{~h}$ light/dark cycle and provided with food and water ad libitum. All animal protocols were in accordance with the NIH Guide for the Care and Use of Laboratory Animals. To assess cancer cell proliferation in vivo, we subcutaneously transplanted ES-2 WT or WISP2 deficient cells $\left(1 \times 10^{6}\right)$ into both back flanks of 8 -week-old female nude mice. Three weeks later, primary tumor masses were collected from athymic nude mice, fixed in $4 \%$ paraformaldehyde, and embedded in paraffin.

\section{Immunohistochemical (IHC) analysis}

Primary tumor masses were excised and fixed in $4 \%$ paraformaldehyde in PBS overnight. For immunochemistry related studies, sections were deparaffinized, rehydrated with xylene and a descending alcohol gradient, and incubated in $0.3 \% \mathrm{H}_{2} \mathrm{O}_{2}$. Following antigen retrieval using $10 \mathrm{mM}$ sodium citrate ( $\mathrm{pH} 6.0$ ), sections were incubated with anti-WISP2, anti-p-ERK1/2, anti-p-YAP, anti-p-Histone $\mathrm{H} 3$, anti-cleaved caspase-3 antibodies (Cell Signaling Technology, 1:200) using a Vector ABC kit (Vector Laboratories) at room temperature for $1 \mathrm{~h}$. Afterward, the sections were allowed to react with biotin-labeled secondary antibodies for $30 \mathrm{~min}$. Staining was performed using the Vectastain $A B C$ kit and 3,3'-diaminobenzidine $(\mathrm{DAB})$ peroxidase substrate kit (Vector Laboratories, Burlingame, CA, USA). 


\section{Immunofluorescence analysis}

Cells were cultured in a 24-well plate overnight, washed with PBS, and fixed for $10 \mathrm{~min}$ at room temperature with $4 \%$ paraformaldehyde in PBS. Cells were permeabilized with $0.3 \%$ Triton X-100 in PBS, incubated with the blocking buffer (PBST containing 5\% bovine serum albumin), and sequentially probed with anti-p-H2AX, anti-Ki-67, and anticleaved caspases-3 antibodies (Cell Signaling Technology, 1: 200) and 488-conjugated secondary antibodies (Molecular Probes). Slides were mounted using a VectaShield with 4', 6-diamidino-2-phenylindole (DAPI, Vector Laboratories). Digital images were acquired using a laser scanning confocal microscope with $6-100 \times$ magnification.

\section{Western blot analysis}

Total proteins were isolated from the cell extracts, and $30 \mu \mathrm{g}$ of protein were separated by SDS-PAGE and transferred to polyvinylidene difluoride (PVDF) membranes (Millipore, Bedford, MA, USA). After probing with primary antibodies, membranes were washed in Tris-buffered saline containing 0.05\% Tween-20 (TBST) and incubated with horse-radish peroxidase-linked secondary antibodies. Finally, the obtained bands were detected using an Enhanced Chemiluminescence Detection Kit (Millipore, Bedford, MA, USA).

The primary antibodies used were as follows:

\begin{tabular}{lll}
\hline Antibodies & Source & Indentifier \\
\hline WISP2 & Abcam & Cat\#:31317 \\
LATS1 & Cell Signaling & Cat\#:3477 \\
YAP & Cell Signaling & Cat\#:14074 \\
YAPS127 & Cell Signaling & Cat\#:4911 \\
LATS2 & Cell Signaling & Cat\#:5888 \\
ERK1/2 & Cell Signaling & Cat\#:4695 \\
p-ERK1/2 & Cell Signaling & Cat\#:4370 \\
Actin & Abcam & Cat\#:ab3280 \\
AKT & Cell Signaling & Cat\#:9272 \\
p-AKT & Cell Signaling & Cat\#:4058 \\
PARP & Cell Signaling & Cat\#:9532 \\
cleaved caspase-3 & Cell Signaling & Cat\#:9664 \\
KI-67 & Cell Signaling & Cat\#:9129 \\
p-Histone H3 & Cell Signaling & Cat\#:9701 \\
\hline
\end{tabular}

\section{RNA extraction and real-time RT-PCR analysis}

Total RNA was extracted using TRIZOL, according to the manufacturer's instructions. Real-time PCR analysis was performed using a KAPA SYBR FAST qPCR kit (Kapa Biosystems, USA) and an Applied 7300 Real-Time PCR System. Relative mRNA levels were determined by normalizing the obtained expression levels to endogenous GAPDH mRNA levels using Microsoft EXCEL. The relative transcript levels of the control sample were set at 1 and compared with the transcript levels of the other samples. Quantitative RT-PCR reactions were performed in triplicate. The following primers were used to amplify target genes:

Gapdh: 5'-GCCTGGAGAAACCTGCCAAGTATG-3' and 5'-GAGTGGGAGTTGCTGTTGAAGTCG-3';

Ctgf: 5'-AGCTGACCTGGAGGAAAACA-3' and 5'GACAGGCTTGGCGATTTTAG-3';

Cyr61: 5' -GCTCAGTCAGAAGGCAGACC-3' and 5'GTTCTTGGGGACACAGAGGA-3';

Amotl2: 5' -AGGAGAAGAGTTGCCCACCTATGAG$3^{\prime}$ and 5' '-TCGAAGAGCTTCATCCTGTCGC-3'; p90RSK: 5'-CAGAGACCTCAAGCCTGAGAAC-3' and $5^{\prime}$-CCACCAGTCCGCACTATGGG-3';

c-Myc: 5'-ACCAGAGTTTCATCTGCGACCC-3' and5'TGGAGGTGGAGCAGACGCTG-3'.

Elk1: 5' -AGGCAATGGCCACATCATCTC-3' and 5' CGCTCCCTTGCGGATGATG-3';

$M d m 2:$ 5'-CCTGGCTCTGTGTGTAATAAG-3' and 5'-ATCCAACCAATCACCTGAATG-3';

Perp: 5'-GGCTTCATCATCCTGGTGAT-3' and 5'ACAGCAGCCAAGGCAAGGAG-3';

Snai1: 5'-AGAGTTTACCTTCCAGCAGCC-3' and 5'-GGACAGAGTCCCAGATGAGC-3';

Vimentin-1: 5'-TACATCGACAAGGTGCGCTT-3' and $5^{\prime}$-TCGTTGGTTAGCTGGTCCAC-3';

Skp2: $5^{\prime}$-TTGCGCATGTGTCAGAGACC-3' and 5'AGGTGTTGGAGGTAGTTGAGC-3';

p16: 5' - CTGCCCAACGCACCGAATAG-3' and 5' ACCACCAGCGTGTCCAGGAA-3' .

Mmp13: 5' - AAGGAGCATGGCGACTTC-3' and 5'TGGCCCAGGAGGAAAAGC-3'.

Htra1: 5'-GGGACTGGTCGTGTTTGTGC-3' and 5' CATTGACCTTTGGGTGCTGACT-3'.

\section{Statistical analyses}

All in vitro assays were performed in triplicate. Groups were compared using two-tailed t-tests or ANOVA via the statistical program GraphPad Prism (GraphPad Prism, San Diego, CA, USA). Statistical significance was set at $p<0.05$.

\section{Abbreviations \\ CEBP: CCAAT/enhancer-binding protein; CRISPR/Cas9: Clustered regularly- interspaced short palindromic repeats/Cas9; CTGF: Connective tissue growth factor; Cyr61: Cysteine-rich 61; DMEM: Dulbecco's Modified Eagle's Medium; DNMT1: DNA methyltransferase 1; ERK: Extracellular signal-related kinase; FACS: Fluorescent-activated cell sorting; LATS: Large tumor suppressor; MAPK: Mitogen-activated protein kinase; MMP: Matrix metalloproteinase; mOSE: Mouse ovarian surface epithelium; Skp2: S-phase kinase-associated protein 2; WISP2: Wnt-inducible signaling protein 2; YAP: Yes-associated protein}

Acknowledgements

We thank Baoqian Shan, Ying Sun, Jingjian Dong, and Lili Shi for technical assistance and Dr. Kun-Liang Guan for the CRISPR/Cas9 plasmid. We also thank Dr. Michal Heger for helpful discussions. 


\section{Authors' contributions}

PW-W made substantial contributions to the conception of the study, performed experiments, interpreted data, and corrected the manuscript. SZ $\mathrm{Q}$ and $\mathrm{CZ}-\mathrm{Y}$ designed the study and wrote the manuscript (first draft). ZY corrected and critically revised the manuscript. HY and ZH analyzed the characteristics of the images and interpreted morphological data. ZH-Y and LM-D performed experiments and wrote specific sections of the manuscript. SZ-Q and PW-W designed the study and participated in the revision of the manuscript. The authors have read and approved the final manuscript.

\section{Authors' information}

The work was performed at the Laboratory of Molecular Biology, College of Medicine, Jiaxing University.

\section{Funding}

This study was supported by grants from the National Natural Science Foundation of China (31871402), the Natural Science Foundation of Zhejiang Province (LY17H160060, LGF2OH160031), and the Student Research Training project (Nos. 202010354043, 202010354042, 851719108, and 851719496) to WWP

\section{Availability of data and materials}

All data generated or analyzed during this study are included in the present article. The datasets supporting the conclusions of this article are included in the article.

\section{Ethics approval and consent to participate}

Human ovarian cancer tissues were provided by the Jiaxing Maternity and Child Health Care Hospital, China. The use of archived samples in this study was approved by the Jiaxing University Institutional Review Board. All animal protocols were in accordance with the NIH Guides for the Care and Use of Laboratory.

\section{Consent for publication}

Not applicable.

\section{Competing interests}

The authors declare no competing interests.

Received: 31 March 2020 Accepted: 15 July 2020

Published online: 25 July 2020

\section{References}

1. Siegel RL, Miller KD, Jemal A. Cancer statistics, 2019. CA Cancer J Clin. 2019; 69(1):7-34.

2. Stewart C, Ralyea C, Lockwood S. Ovarian cancer: an integrated review. Semin Oncol Nurs. 2019;35(2):151-6.

3. Brigstock DR, Goldschmeding R, Katsube Kl, Lam SC, Lau LF, Lyons K, et al. Proposal for a unified CCN nomenclature. Mol Pathol. 2003;56:127-8.

4. Leask A, Abraham DJ. All in the CCN family: essential matricellular signaling modulators emerge from the bunker. J Cell Sci. 2006;119:4803-10.

5. Perbal B. CCN proteins: a centralized communication network. J Cell Commun Signal. 2013;7:169-77.

6. Li J, Ye L, Owen S, Weeks HP, Zhang Z, Jiang WG. Emerging role of CCN family proteins in tumorigenesis and cancer metastasis. Int J Mol Med. 2015; 36:1451-63.

7. Inadera $\mathrm{H}$, Shimomura A, Tachibana S. Effect of Wnt-1 inducible signaling pathway protein-2 (WISP-2/CCN5), a downstream protein of Wnt signaling, on adipocyte differentiation. Biochem Biophys Res Commun. 2009;379:96974.

8. Russo JW, Castellot JJ. CCN5: biology and pathophysiology. J Cell Commun Signal. 2010;4:119-30.

9. Berger T, Sidhu P, Tang S, Kucera H. Are testicular cortisol and WISP2 involved in estrogen-regulated Sertoli cell proliferation? Anim Reprod Sci. 2019;207:44-51.

10. Pan WW, Moroishi T, Koo JH, Guan KL. Cell type-dependent function of LATS1/2 in cancer cell growth. Oncogene. 2019;38(14):2595-610.

11. Sharpless NE, Sherr CJ. Forging a signature of in vivo senescence. Nat Rev Cancer. 2015;15:397-08.
12. Frewer KA, Sanders AJ, Owen S, Frewer NC, Hargest R, Jiang WG. A role for WISP2 in colorectal cancer cell invasion and motility. Cancer Genomics Proteomics. 2013;10:187-96.

13. Dhar G, Mehta S, Banerjee S, Gardner A, McCarty BM, Mathur SC, et al. Loss of WISP-2/CCN5 signaling in human pancreatic cancer: a potential mechanism for epithelial-mesenchymal-transition. Cancer Lett. 2007;254:6370 .

14. Ji J, Jia S, Jia Y, Ji K, Hargest R, Jiang WG. WISP-2 in human gastric cancer and its potential metastatic suppressor role in gastric cancer cells mediated by JNK and PLC- $\gamma$ pathways. Br J Cancer. 2015;113:921-33.

15. Chai DM, Qin YZ, Wu SW, Ma L, Tan YY, Yong X, et al. WISP2 exhibits its potential antitumor activity via targeting ERK and E-cadherin pathways in esophageal cancer cells. J Exp Clin Cancer Res. 2019:38:102-17.

16. Inadera H, Hashimoto S, Dong HY, Suzuki T, Nagai S, Yamashita T, et al. WISP-2 as a novel estrogen-responsive gene in human breast cancer cells. Biochem Biophys Res Commun. 2000;275:108-14.

17. Ray G, Banerjee S, Saxena NK, Campbell DR, Van Veldhuizen P, Banerjee SK Stimulation of MCF-7 tumor progression in athymic nude mice by 17 betaestradiol induces WISP-2/CCN5 expression in xenografts: a novel signaling molecule in hormonal carcinogenesis. Oncol Rep. 2005;13:445-8.

18. Haque I, Banerjee S, De A, Maity G, Sarkar S, Majumdar M, et al. CCN5/WISP2 promotes growth arrest of triple-negative breast cancer cells through accumulation and trafficking of p27(kip1) via Skp2 and FOXO3a regulation. Oncogene. 2015:34:3152-63.

19. Yu P, Ye L, Wang H, Du G, Zhang J, Zhang J, et al. NSK-01105 inhibits proliferation and induces apoptosis of prostate cancer cells by blocking the Raf/MEK/ERK and PI3K/Akt/mTOR signal pathways. Tumour Biol. 2015;36: 2143-53.

20. Yu FX, Zhao B, Guan KL. Hippo pathway in organ size control, tissue homeostasis, and cancer. Cell. 2015;163:811-28.

21. Liu S, Zha J, Lei M. Inhibiting ERK/Mnk/elF4E broadly sensitizes ovarian cancer response to chemotherapy. Clin Transl Oncol. 2018;20:374-81.

22. Hu Y, Yang L, Yang $Y$, Han $Y$, Wang $Y$, Liu W, et al. Oncogenic role of mortalin contributes to ovarian tumorigenesis by activating the MAPK-ERK pathway. J Cell Mol Med. 2016:20:2111-21.

23. Dang JH, Jin ZJ, Liu XJ, Hu D, Wang J, Luo Y, et al. Metformin in combination with cisplatin inhibits cell viability and induces apoptosis of human ovarian cancer cells by inactivating ERK 1/2. Oncol Lett. 2017;14: 7557-64.

24. Xia Y, Chang T, Wang Y, Liu Y, Li W, Li M, et al. YAP promotes ovarian cancer cell tumorigenesis and is indicative of a poor prognosis for ovarian cancer patients. PLoS One. 2014;9:e91770.

25. Liu SB, Lin XP, Xu Y, Shen ZF, Pan WW. DAXX promotes ovarian cancer ascites cell proliferation and migration by activating the ERK signaling pathway. J Ovarian Res. 2018;11:90

26. Barreto SC, Ray A, Ag EP. Biological characteristics of CCN proteins in tumor development. J BUON. 2016;21:1359-67.

27. Prieto-García E, Díaz-García CV, García-Ruiz I, Agulló-Ortuño MT. Epithelial-tomesenchymal transition in tumor progression. Med Oncol. 2017;34:122.

28. Wong SHM, Fang CM, Chuah LH, Leong CO, Ngai SC. E-cadherin: its dysregulation in carcinogenesis and clinical implications. Crit Rev Oncol Hematol. 2018;121:11-22.

29. Banerjee SK, Banerjee S. CCN5/WISP-2: a micromanager of breast cancer progression. J Cell Commun Signal. 2012;6:63-71.

\section{Publisher's Note}

Springer Nature remains neutral with regard to jurisdictional claims in published maps and institutional affiliations. 